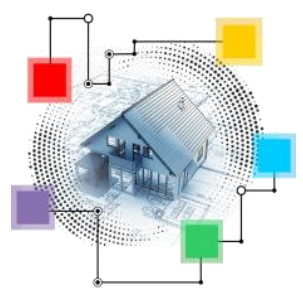

\author{
XIII SIMPÓSIO NACIONAL DE SISTEMAS PREDIAIS \\ DESEMPENHO E INOVAÇÃO \\ DE SISTEMAS PREDIAIS HIDRÁULICOS \\ SÃO PAULO - 04 DE OUTURO DE 2019
}

\title{
Mapeamento das principais causas de contaminação de água potável em sistemas prediais hidrossanitários \\ Mapping of main causes of potable water contamination in predial hydrosanitary systems
}

\author{
OLIVEIRA, Paulo Henrique Stehling'; BARRETO, Douglas 2
}

1. Universidade Federal de São Carlos - Engenheiro Civil, Discente do Programa de Pós-Graduação em Engenharia Civil do Departamento de Engenharia Civil e Docente da UNASP, Est. Municipal Pr. Walter Boger, s/n - Engenheiro Coelho, paulo.stehling@ucb.org.br

2. Universidade Federal de São Carlos - Docente no Programa de Pós-Graduação em Engenharia Civil do Departamento de Engenharia Civil, dbarreto@ufscar.br

\begin{abstract}
RESUMO
Dentre os sistemas prediais o que mais tem potencial para apresentar manifestações patológicas é o sistema hidrossanitário. Existem diversas manifestações patológicas possíveis e uma delas é a contaminação da água potável no sistema de distribuição e armazenamento. Em algumas situações a água apresenta adequado padrão de potabilidade na fonte, mas no reservatório encontra-se contaminada. O método adotado consistiu de uma revisão bibliográfica, cujo objetivo foi mapear, identificar e classificar as principais causas de contaminação de água potável em sistemas prediais hidrossanitários em diversas bases de dados. Como resultado, foram identificadas diversas causas de contaminação, e estas foram classificadas em: conexão cruzada; desgaste de materiais; tempo de armazenamento; manutenção; vedação; equipamentos; projeto; vandalismo/sabotagem; tratamento inadequado; e fonte. Pode-se concluir que as causas são diversas e que existem medidas a serem tomadas para evitá-las, e entre as recomendações pode-se concluir que há a necessidade de implantar uma cultura de manutenção e que o uso de reservatórios enseja muitos cuidados para garantir a qualidade da água no sistema de distribuição e consumo. Por fim, ressalta-se a importância de um projeto dos sistemas prediais hidrossanitários bem elaborado e atendendo as recomendações técnicas.
\end{abstract}

Palavras-chave: Sistemas prediais; instalações hidráulicas; qualidade de água; contaminação; reservatórios.

\begin{abstract}
Among the building services systems, the one with the potential to present pathological manifestations is the hydrosanitary system. There are several possible pathological manifestations and one of them is the contamination of drinking water in the distribution and storage system. In some situations, the water has an adequate standard of potability at the source, but in the reservoir, it is contaminated. The method adopted consisted of a bibliographic review, whose objective was to map, identify and classify the main causes of contamination of drinking water in hydrosanitary land systems in several databases. As a result, the several causes of contamination identified and classified were: crossconnection; wear of materials; storage time; maintenance; seal; equipment; project; vandalism/sabotage; inadequate treatment; and source. It can be concluded that the causes are diverse and that there are measures to be taken to avoid them, and among the recommendations it can be concluded that there is a need to implement a culture of maintenance and that the use of reservoirs requires much care to ensure the quality of water in the distribution and consumption system. Finally, the importance of a well-developed hydrosanitary systems project is emphasized and taking into account the technical recommendations.
\end{abstract}

Keywords: Building services systems; Hidraulic systems; water quality; contamination; reservoirs. 


\section{INTRODUÇÃO}

Um dos sistemas prediais que mais provocam manifestações patológicas nas edificações são os hidrossanitários. Quando as instalações são enterradas se torna mais difícil a detecção de anomalias, gerando um problema silencioso e com grande potencial destrutivo. No caso de vazamento em tubulações enterradas de esgoto sanitário, o material que infiltra no solo traz um alto nível de contaminação, podendo prejudicar grandemente o sistema de alimentação de água potável.

Segundo a NBR 5626:1998 (ABNT, 1998) “os reservatórios de água potável constituem uma parte crítica da instalação predial de água fria no que diz respeito à manutenção do padrão de potabilidade".

A água pode estar com a qualidade adequada na fonte, mas não estar no reservatório, segundo Santos (2002) “o fato da água encontrar-se potável na entrada da edificação não garante que a mesma esteja potável no ponto de consumo. Isto ocorre porque existem pontos potenciais de contaminação de água no referido trajeto".

A causa inicial da contaminação da rede pode ser por material de esgotamento sanitário que contamina o solo e, devido a falhas também na tubulação de água fria, contamina a água tornando-a inadequada ao consumo.

Geldreich (1996) citado por Moreno (2009) afirma que "a qualidade da água pode ser degradada no sistema distribuidor pois, em seu caminho entre a unidade de tratamento e a torneira do consumidor ocorrem reações químicas e biológicas que estão relacionadas.

Reali et al. (2002) aponta que "a localização imprópria do reservatório, a ignorância do usuário em relação à conservação do reservatório, a falta de cobertura adequada e a ausência de limpezas periódicas são os principais fatores que contribuem para a alteração da qualidade da água”.

O objetivo geral deste trabalho é apresentar e discutir os resultados do mapeamento das principais causas de contaminação de água potável em sistemas prediais hidrossanitários.

\section{MATERIAIS E MÉTODOS}

Este trabalho consistiu em uma Revisão Bibliográfica Sistemática, tendo como base o "BS Roadmap" proposto por Conforto E.C.; Amaral D. C.; Silva S.L (2011) apresentado na Figura 1, a seguir.

A bases de pesquisa foram: Portal de Periódicos - Capes, ScienceDirect, Scielo, Biblioteca Digital de Teses e Dissertações, todas de livre acesso.

Como palavras-chave para busca nas bases dados foram: building systems; plumbing system; waste pipe; buried pipes; underground piping; pathological manifestations; water tank; reservoir; vessel; contamination; quality; drinking water; water pollution building; water quality; building water supply; distribution system; domestic drinking water; deterioration; household; private water storage tank.

Foram estabelecidos os seguintes critérios para definição dos artigos a serem analisados na RBS: ano de publicação; local de estudo; tipo de contaminação e etapa de contaminação. 
FIGURA 1- Processo da RBS roadmap.

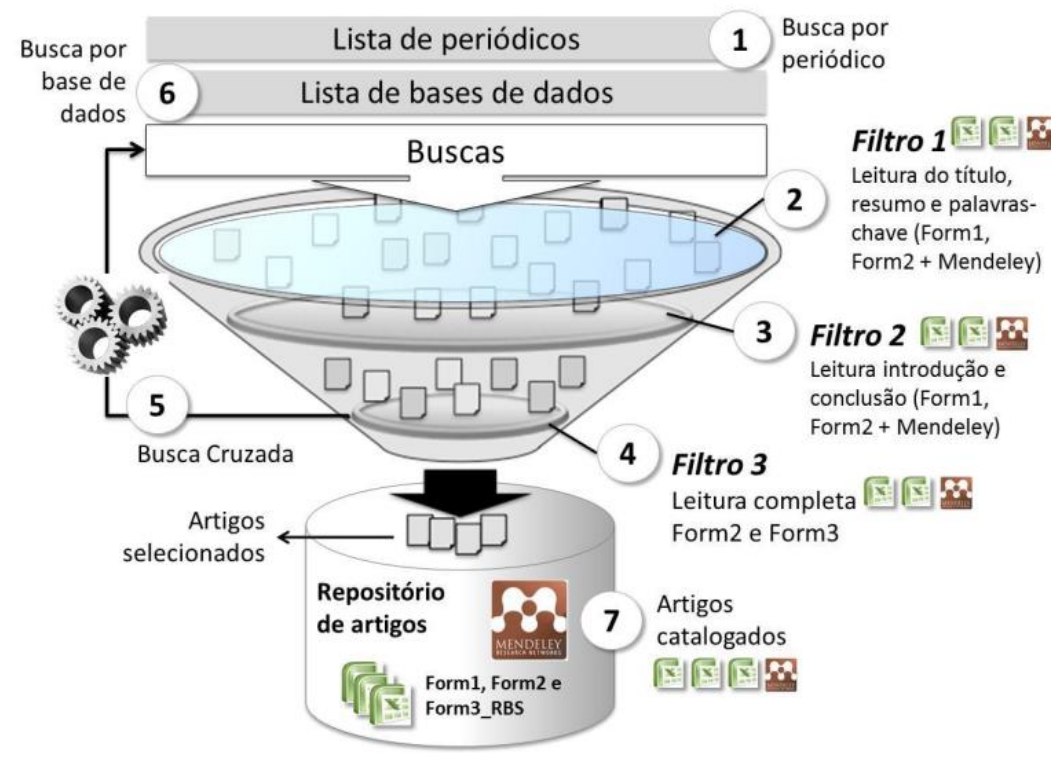

Fonte: Conforto et.al.(2011)

\section{RESULTADOS E ANÁLISES}

A seguir serão apresentados os resultados obtidos e suas respectivas análises do mapeamento sistemático realizado.

\subsection{Mapeamento}

Foi realizada uma pesquisa em bases de dados com as seguintes palavras-chave: building systems; plumbing system; waste pipe; buried pipes; underground piping; pathological manifestations; water tank; reservoir; vessel; contamination; quality; drinking water; water pollution building; water quality; building water supply; distribution system; domestic drinking water; deterioration; household; private water storage tank. Estas palavras foram utilizadas com diversas combinações entre si, resultando em 36 documentos analisados, sendo 29 artigos; 2 Normas, 3 teses e 2 apostilas.

Foi realizada uma depuração nas referências encontradas, considerando a relevância e convergência do teor dos documentos e com o material selecionado foi feita uma compilação considerando: o ano de publicação; o país ou região do local de estudo; o tipo de estudo realizado; o tipo de contaminação; e por etapa de contaminação; As Figuras 2 a 6 a seguir apresentam os resultados das classificações. 
FIGURA 2 - Classificação dos artigos por ano de publicação.

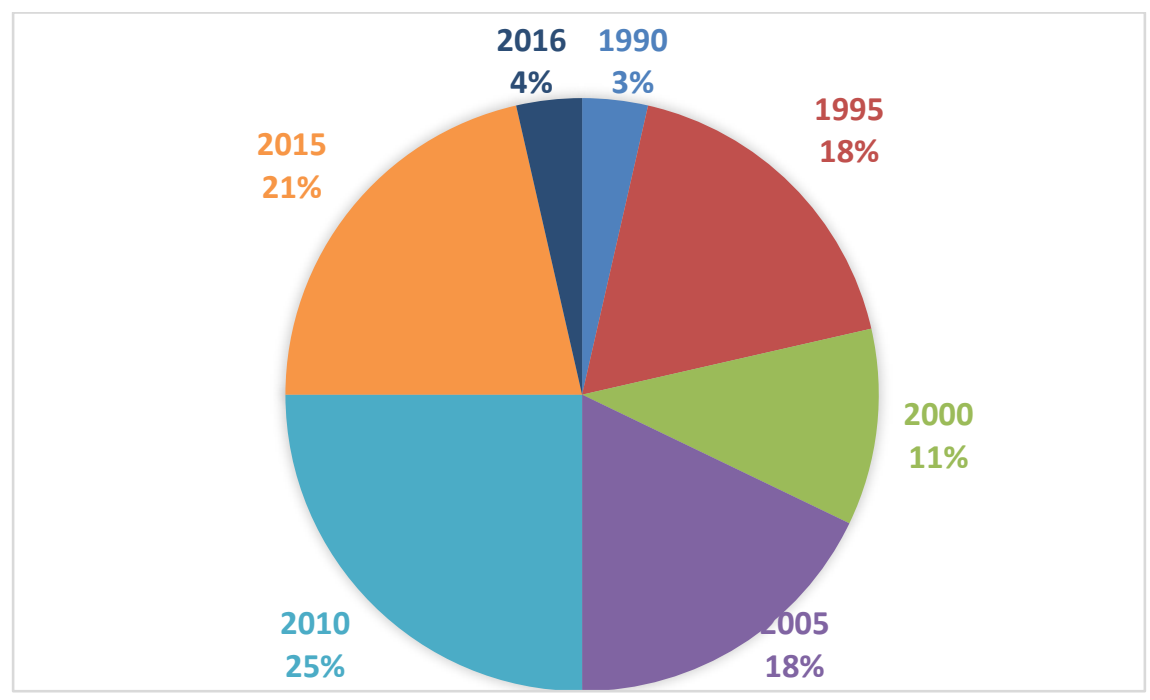

Fonte: Autores.

FIGURA 3 - Classificação dos artigos por país ou região do local de estudo.

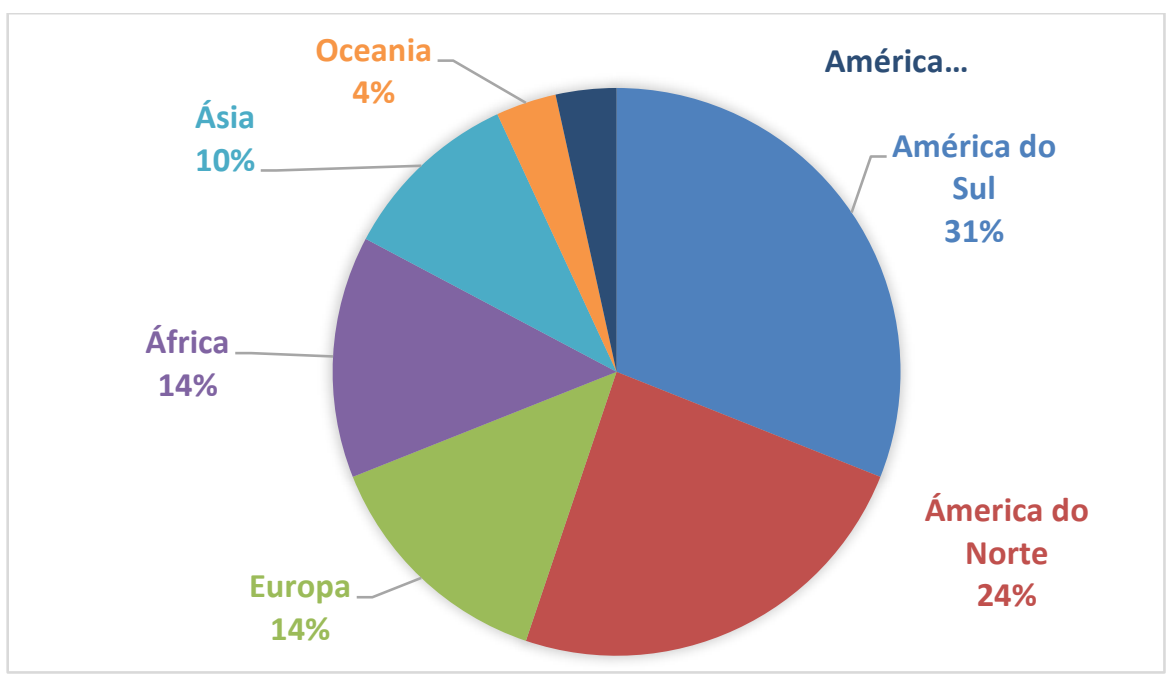

Fonte: Autores.

FIGURA 4 - Classificação dos artigos por tipo de pesquisa.

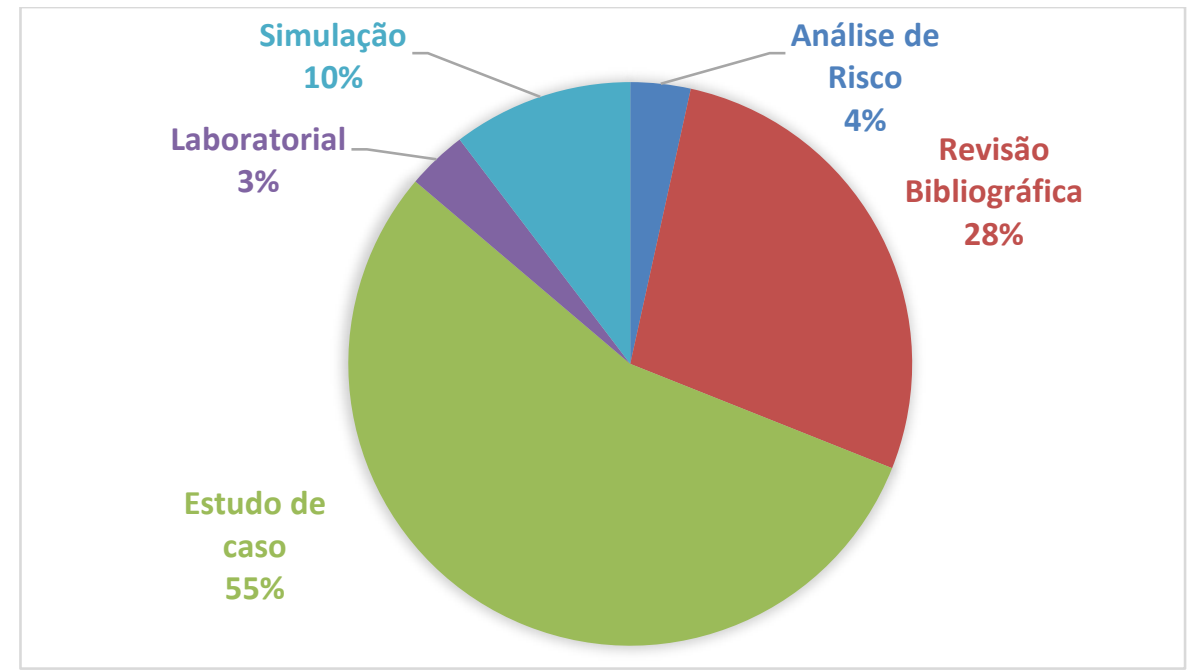

Fonte: Autores. 
FIGURA 5 - Classificação dos artigos por tipo de contaminação.

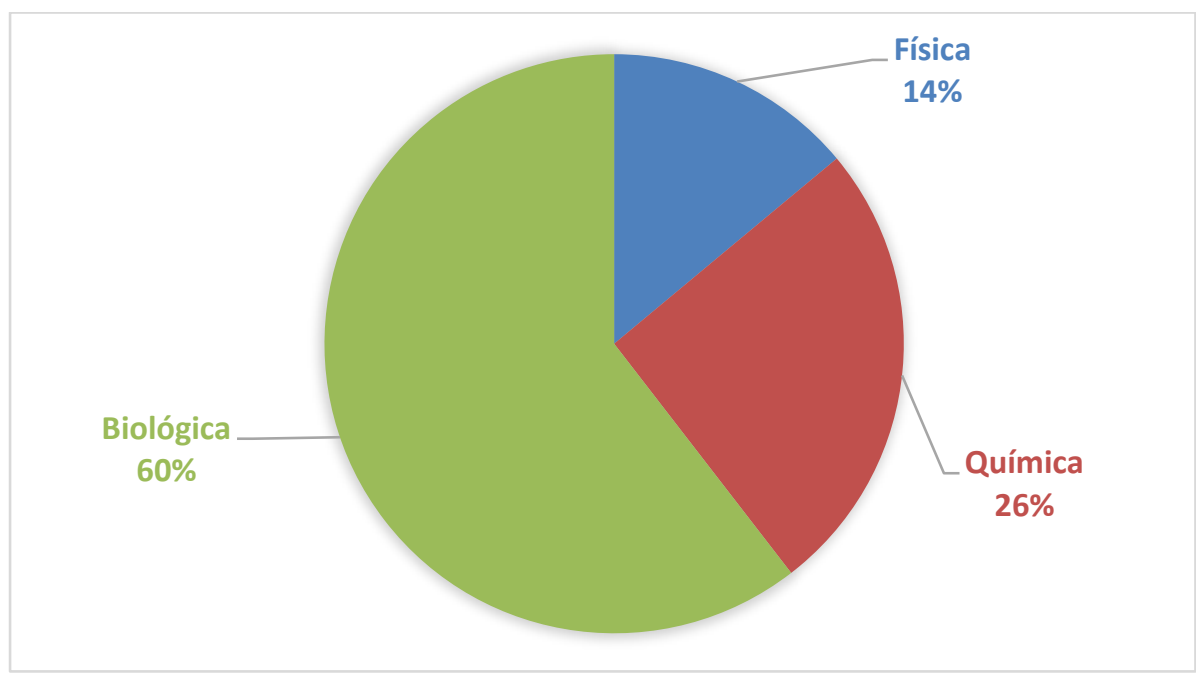

Fonte: Autores.

FIGURA 6 - Classificação dos artigos por etapa de contaminação.

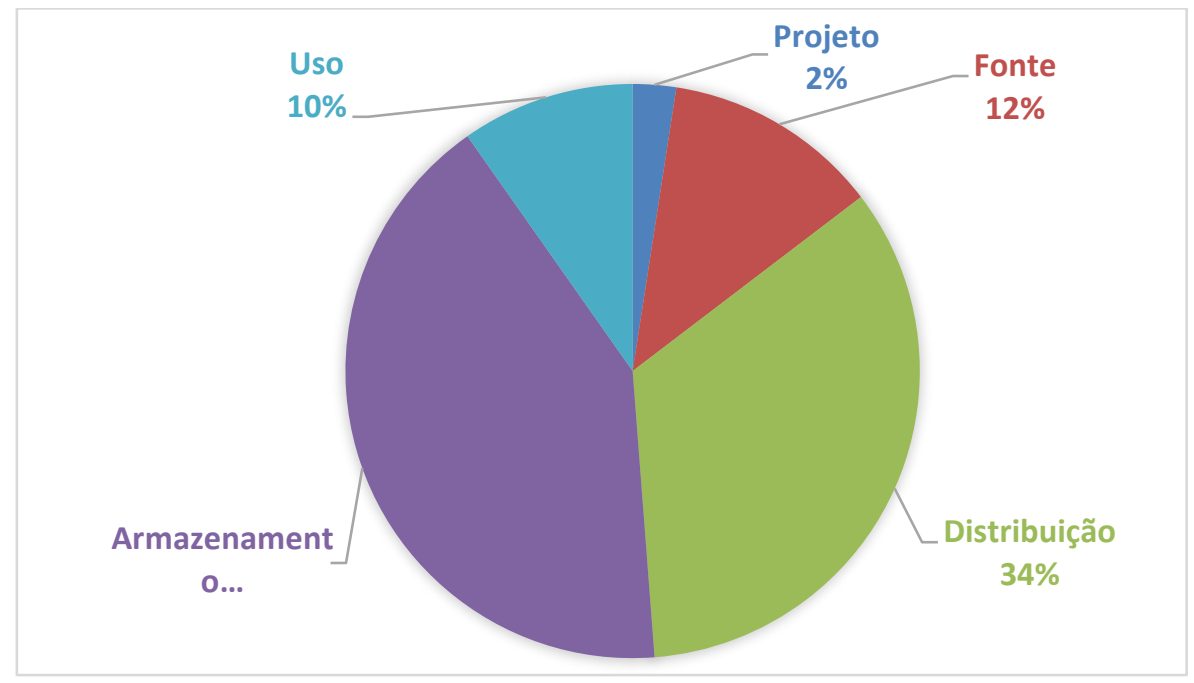

Fonte: Autores.

A respeito do mapeamento realizado verificou-se que no período da pesquisa realizada a concentração de artigos data de 2005 a 2015. Em relação aos países ou locais a maioria se concentra nas Américas Sul e do Norte, denotando-se que estas regiões têm ocorrido manifestações de contaminação com maior frequência. A maioria dos tipos de pesquisa verificado são Estudos de Caso, que podem indicar alta frequência da ocorrência de contaminações. Quanto ao tipo de contaminação verifica-se que a maioria foi biológica seguida por contaminação química. Quanto à origem verificou-se que se concentra em duas etapas, de distribuição e de armazenamento, ou seja, na rede e nos reservatórios das residências.

\subsection{Classificação}

A partir dos artigos selecionados foi possível classificar as causas de contaminação nas seguintes categorias: Conexão cruzada; Desgaste de materiais; Tempo de armazenamento; Manutenção; Vedação; Equipamentos; Projeto; Vandalismo/sabotagem. 


\subsubsection{Conexão cruzada}

Segundo Zacarias e Bulbo (1987) citado por Tomaz (2010) a conexão cruzada "é possível devido ao contato de águas servidas com a água potável da rede. O ponto onde este contato pode vir a ocorrer denomina-se conexão cruzada ou Cross-Connection”.

A conexão cruzada pode ser dividida entre 'direta' e 'indireta'. Segundo Tomaz (2010) "a conexão cruzada direta é aquela que permite o fluxo da água de um sistema para outro, simplesmente existindo uma pressão diferencial entre os dois”. A Figura 7 ilustra este tipo de fenômeno.

FIGURA 7 - Exemplo de conexão cruzada

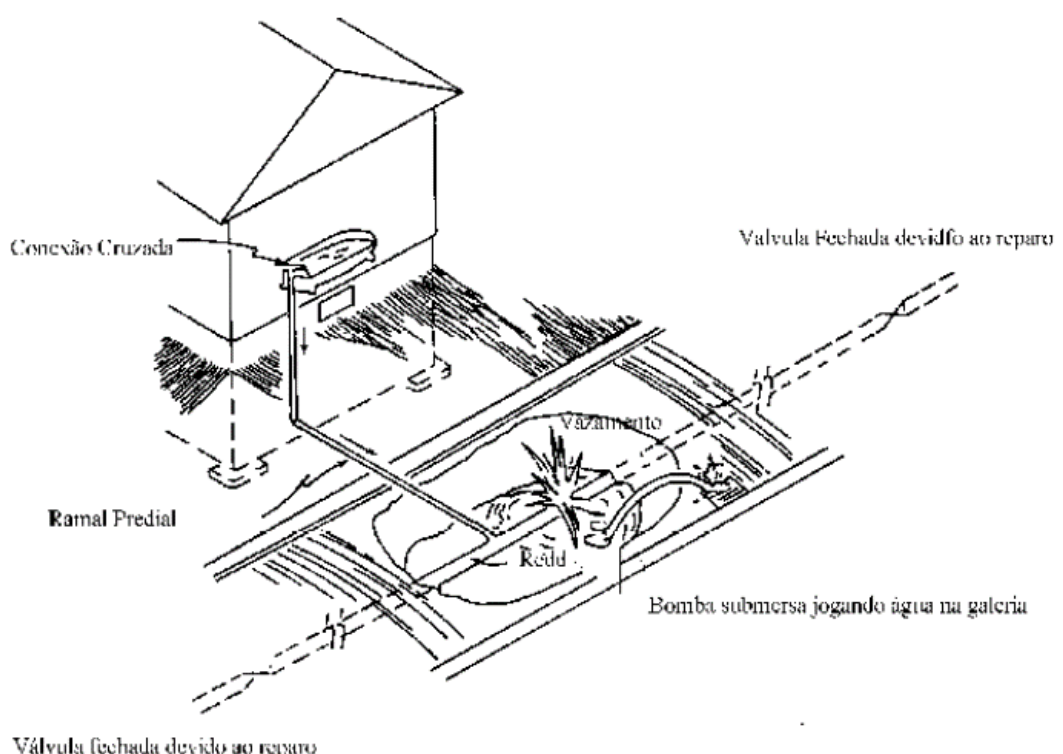

Fonte: Tomaz (2010) [5]

No caso da conexão cruzada indireta Tomaz (2010) afirma que ela acontece quando "o fluxo de água no sentido da rede está sujeito a uma situação anormal, que venha aproximar a água servida o suficiente da extremidade do ponto de utilização para que esta possa ser succionada para a tubulação", denominada de "retrossifonagem".

De acordo com Reali et al (2002) "a retrossifonagem pode ocorrer em aparelhos que apresentam a entrada de água potável abaixo do plano de transbordamento dos mesmos." A Figura 8 mostra esta diferença de níveis em um lavatório.

FIGURA 8 - Níveis de entrada e transbordamento de lavatório.

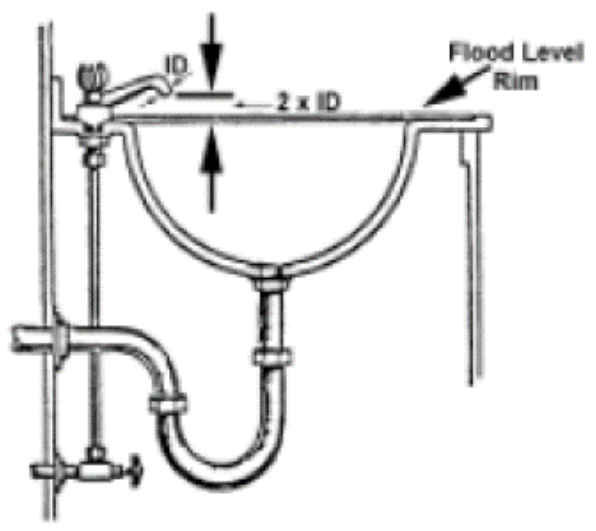

Fonte: Tomaz, 2010. 
Uma observação importante é que nos dois casos de conexão cruzada deve haver uma queda de pressão na rede para induzir o refluxo da água. Por este motivo, situações que levem a pressões negativas sempre devem ser evitadas.

LeChevallier (2003) citado por Moreno (2009) afirma que "os organismos fecais e vírus humanos podem estar presentes na água subterrânea próxima à tubulação e durante transientes de baixa ou negativa pressão poderão ser arrastados para dentro das tubulações". A Figura 9 ilustra este fenômeno com simulação realizada em laboratório.

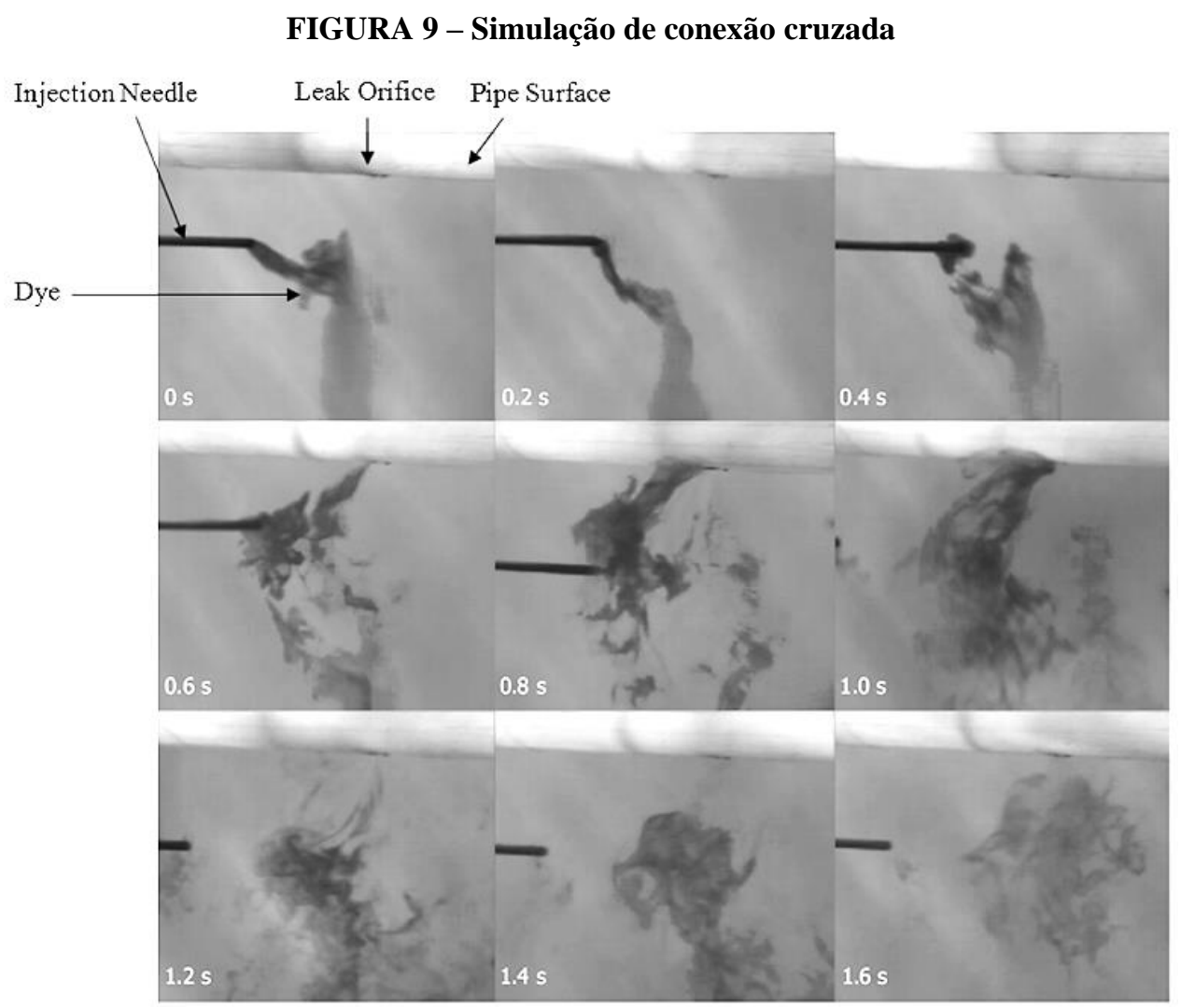

Fonte: Fox et al (2016)

\subsubsection{Desgaste de materiais}

Alguns artigos apresentam o desgaste dos materiais componentes do sistema com uma das causas de contaminação. Segundo Lee e Schwab (2005) a corrosão é um fator essencial no processo de envelhecimento natural.

A corrosão é um meio de introdução de material orgânico e inorgânico no sistema de fornecimento de água, e há evidências que a corrosão afeta a qualidade da água nos sistemas de distribuição. (LEE e SCHAWB, 2005). A Figura 10, a seguir, ilustra um exemplo de corrosão em tubulação. 
FIGURA 10 - Corrosão em tubo

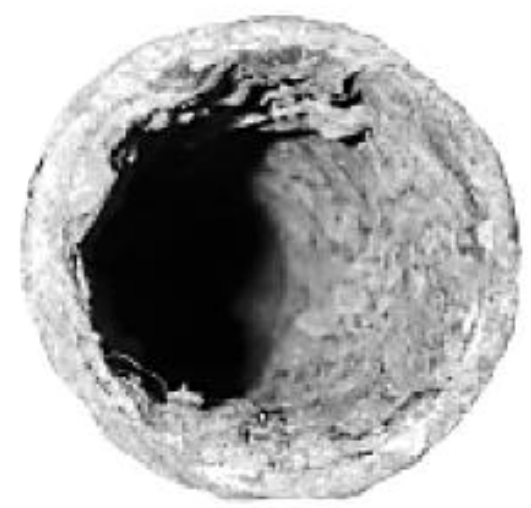

Fonte: Lahlou (2002)

\subsubsection{Tempo de armazenamento}

A idade da água é um fator importante na deterioração da qualidade da água em um sistema de distribuição. “À medida que a água viaja através do sistema de distribuição, ela sofre várias transformações químicas, físicas e estéticas, impactando a qualidade da água”. (AWWA, 2002).

Como alerta importante aos projetistas de sistemas prediais Moreno (2009) afirma que "o sistema de distribuição não deve ter capacidade excessiva, pois resultarão longos tempos de trânsito da água entre a unidade de tratamento e o consumidor". O Quadro 1, a seguir, mostra quais são os principais problemas associados com a idade da água.

QUADRO 1 - Problemas associados com a idade da água

\begin{tabular}{|c|c|c|}
\hline Problemas químicos & Problemas Biológicos & Problemas Físicos \\
\hline $\begin{array}{l}\text { *Desinfecção por } \\
\text { formação produtos }\end{array}$ & $\begin{array}{c}\text { *desinfecção por biodegradação } \\
\text { de produto }\end{array}$ & Elevação da temperatura \\
\hline $\begin{array}{l}\text { Decaimento de } \\
\text { desinfecção }\end{array}$ & *Nitrificação & Deposição de sedimentos \\
\hline $\begin{array}{c}\text { *Efetividade no controle } \\
\text { da corrosão }\end{array}$ & $\begin{array}{l}* \text { Crescimento microbiano/ } \\
\text { recobrimento/cobrimento }\end{array}$ & cor \\
\hline Sabor e odor & Sabor e odor & \\
\hline
\end{tabular}

Fonte: Livre tradução de AWWA (2002)

Segundo a NBR 5626 (1998) “os reservatórios destinados a armazenar água potável devem preservar o padrão de potabilidade. Em especial não devem transmitir gosto, cor, odor ou toxicidade à água nem promover ou estimular o crescimento de microrganismos".

\subsubsection{Manutenção}

Uma causa de contaminação importante no contexto de países em desenvolvimento, como é o caso do Brasil, é ausência de manutenção adequada nos reservatórios. A recomendação técnica é que eles recebam limpeza a cada 6 meses, mas na maioria dos casos esta medida não é cumprida. A limpeza periódica dos reservatórios elimina a presença do biofilme que pode se formar. 
Biofilme, segundo Julião (2011), "é um complexo ecossistema microbiológico e a dinâmica da sua formação ocorre em etapas distintas, iniciando-se com a adesão dos organismos colonizadores a uma superfície, geralmente contendo proteínas ou outros compostos orgânicos".

Reali et al (2002) afirma que "a existência de uma camada de matéria orgânica e inorgânica no fundo do reservatório provoca um aumento da turbidez e cor, é responsável pelo consumo da maior parte do cloro residual da água afluente e acarreta a diminuição do oxigênio dissolvido". A Figura 11 mostra como funciona o desenvolvimento de um biofilme.

FIGURA 11 - Desenvolvimento de um biofilme.

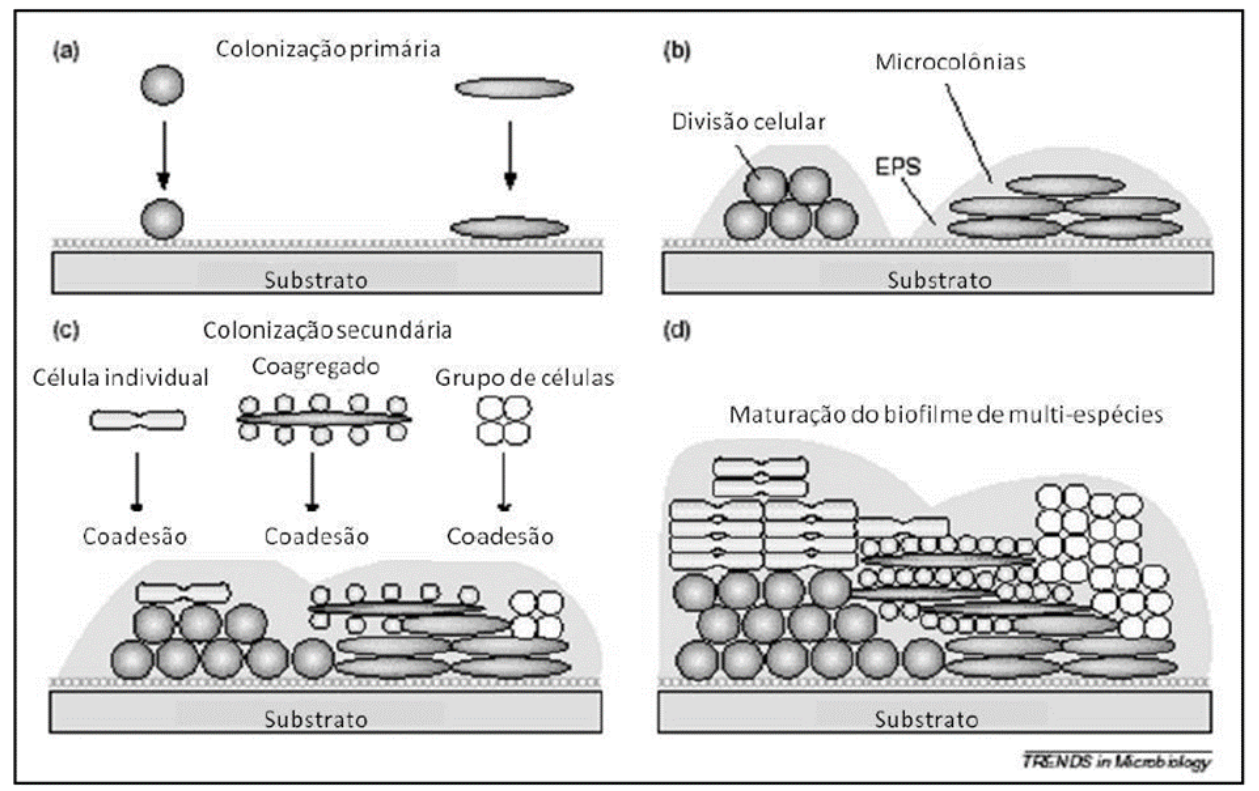

Fonte: Julião (2011)

\subsubsection{Vedação}

A falta de vedação adequada nos reservatórios também se mostrou uma das principais causas de contaminação. Esta pode ocorrer tanto pelo contato direto de animais e suas fezes, quanto pelo contato direto dos usuários.

A NBR 5626 (ABNT, 1998) preconiza que "o reservatório deve ser um recipiente estanque que possua tampa ou porta de acesso opaca, firmemente presa na sua posição, com vedação que impeça a entrada de líquidos, poeiras, insetos e outros animais no seu interior".

\subsubsection{Equipamentos}

Alguns trabalhos apontaram os filtros como um dos principais causadores de contaminação da água potável. A explicação fornecida por Scuracchio (2010) é que "esses filtros geralmente retiram o cloro da água e, quando não substituídos e higienizados adequadamente, favorecem a proliferação de microrganismos".

A conclusão de Scuracchio (2010) foi que "os filtros foram os maiores responsáveis pela depreciação da qualidade da água, em relação ao aumento de bactérias heterotróficas e redução do teor de cloro, aumentando, portanto, o risco de doenças". 


\subsubsection{Projeto}

A respeito das falhas de projeto que podem causar contaminação de água, Gnipper (1993) citado por Gnipper e Mikaldo (2007) afirma que "os vícios podem ocorrer por falhas de concepção sistêmica, erros de dimensionamento, ausência ou incorreções de especificações de materiais e de serviços, insuficiência ou inexistência de detalhes construtivos, etc".

Gnipper e Mikaldo (2007) apresentam uma tabela que reúne as principais manifestações patológicas que podem ocorrer em decorrência de inconformidades na fase de projeto.

\subsubsection{Vandalismo/sabotagem}

Uma causa menos recorrente, porém importante, é a contaminação por ação humana proposital e intencional. Podem ocorrer situações em que o sistema de água potável encontra-se vulnerável para a ação de terceiros, podendo sofrer ações de vandalismo ou sabotagem.

Pensando nesta situação torna-se necessário abordar o tema da segurança dos recursos hídricos. "A melhor defesa contra a sabotagem é o planejamento preventivo para a segurança da infraestrutura das redes de distribuição da água”. (Tularam e Properjohn, 2010)

\section{CONSIDERAÇÕES GERAIS}

A primeira conclusão tirada a partir desta pesquisa é que existem diversas e variadas causas de contaminação de água potável em sistemas prediais hidrossanitários. São inúmeros fatores causadores de contaminação, tornando-se até complicada a tarefa de classificá-los.

Pode-se observar também que este é um problema muito comum e recorrente, principalmente em países em desenvolvimento. Isto se deve ao fato que nestes países o regime de abastecimento de água potável normalmente é feito de forma indireta ou mista, devido a intermitência do abastecimento.

Com isto, conclui-se que o sistema indireto, com uso de reservatórios prediais, aumenta as possibilidades de contaminação da água. Então, o uso de reservatórios deve ser evitado, se possível.

Observa-se também uma necessidade prevalente de implantar uma cultura de manutenção em países como o Brasil. Com medidas simples de limpeza e manutenção muitos problemas de saúde podem ser evitados.

Pode-se apontar também que existe abertura no mercado para desenvolvimento de novos materiais componentes dos sistemas prediais. Novos materiais, com menor nível de contaminação, podem proporcionar maior durabilidade da qualidade da água no sistema de distribuição.

Por último, ressalta-se a importância de os profissionais de projeto de sistemas prediais apresentarem projetos bem dimensionados, adequados às normas e às recomendações técnicas, como sendo uma ação preventiva no sentido de eliminar falhas nos sistemas e resultando num projeto de qualidade e de fácil execução. 


\section{REFERÊNCIAS}

ASSOCIAÇÃO BRASILEIRA DE NORMAS TÉCNICAS. NBR 5626. Instalação predial de água fria. Rio de Janeiro: ABNT, 1998.

AWWA (American Water Works Association) and Economic and Engineering Services. Effects of water age on distribution system water quality. Prepared for: U.S. Environmental Protection Agency. Washington, 2002.

BERARDI, L.; GIUSTOLISI, O.; KAPELAN, Z.; SAVIC, D. A. Development of pipe deterioration models for water distribution systems using EPR. Journal of Hydroinformatics, 10.2, 2008, p.113-126.

CAMILOTTI, F. E. G.; GONÇALVES, M. L. Avaliação do sistema de armazenamento de água em edificações. IX Encontro Nacional de Tecnologia do Ambiente Construído. Foz do Iguaçu, 2002.

CAMPOS, J. A. D. B.; FARACHE FILHO, A.; FARIA, J. B. Qualidade da água armazenada em reservatórios domiciliares: parâmetros físico-químicos e microbiológicos. Alim. Nutr., Araraquara, v.14, n.1, pp. 63-67, 2003.

CLARK, R. M.; GRAYMAN, W. M.; MALES, R. M.; HESS, A. F. Modeling contaminant propagation in drinking-water distribution systems. J. Environ. Eng. 1993.119:349-364.

CONFORTO E.C.; AMARAL D. C.; SILVA S.L. Roteiro para revisão bibliográfica sistemática: aplicação no desenvolvimento de produtos e gerenciamento de projetos. $8^{\circ}$ Congresso Brasileiro de Gestão de Desenvolvimento de Produto. Porto Alegre - RS, Set. 2011.

ERCUMEN, A.; GRUBER, J. S.; COLFORD JR., J. M. Water Distribution System Deficiencies and Gastrointestinal Illness: A Systematic Review and Meta-Analysis. Environmental Health Perspectives, vol. 122, n. 7, July 2014.

ESHCOL, J.; MAHAPATRA, P.; KESHAPAGU, S. Is fecal contamination of drinking water after collection associated with household water handling and hygiene practices? A study of urban slum households in Hyderabad, India. Journal of Water and Health, 07.1, pp. 145-154, 2009.

FOX, S.; SHEPERD, W.; COLLINS, R.; BOXALL, J. Experimental quantification of contaminant ingress into a buried leaking pipe during transient events. Journal of Hydraulic Engineering, 142(1), 2016.

FREIRE, R. C. Qualidade da água nos reservatórios domiciliares na região metropolitana da cidade do Recife, Pernambuco. J Manag Prim Health Care 2012; 3(2):102-105.

GAMAL, H. F.; RASHED, G. E. A.; SARAWY, A. A. Microbiological Quality of Potable Water in Distribution Systems from Small Units in Dakahlia Governorate. Fourth International Water Technology Conference IWTC 99, Alexandria, Egypt.

GIBBS, S. G.; MECKES, M. C.; SCARPINO, P. V. The effect of long-term wastewater crossconection on the biofilm of a simulated water distribution system. Journal of Environmental Engineering and Science; Mar 2003; 2, 2; pp.85-98. 
GNIPPER, S. F.; MIKALDO JR, J. Patologias frequentes em sistemas prediais hidráulicosanitários e de gás combustível decorrentes de falhas no processo de produção do projeto. Anais do VII workshop brasileiro de gestão do processo de projetos na construção de edifícios. Universidade Federal do Paraná, Curitiba, 2007.

GUNDRY, S. W. Contamination of drinking water between source and point-of-use in rural households of South Africa and Zimbabwe: implications for monitoring the Millennium Development Goal for water. Water Practice \& Technology Vol 1 No 2, 2006.

JULIÃO, F. C. Avaliação das condições microbiológicas e físico-químicas da água de reservatório domiciliar e predial: importância da qualidade dessa água no contexto da saúde pública. Tese (Doutorado) - Escola de Enfermagem de Ribeirão Preto da Universidade de São Paulo, Ribeirão Preto, 2011.

LAHLOU, Z. M. Water quality in distribution systems. A national drinking water clearinghouse fact sheet, p. 1-4. Morgantown, 2002.

LEE, Y. An Evaluation of Microbial and Chemical Contamination Sources Related to the Deterioration of Tap Water Quality in the Household Water Supply System. International Journal of Environmental Research and Public Health, 2013, 10, 4143-4160.

LEE, E. J.; SCHWAB, K. J. Deficiencies in drinking water distribution systems in developing countries. Journal of Water and Health, 3.2, p. 109-127, 2005.

MIRANDA, C. A. S.; MONTEIRO, T. C. N. Qualidade de água em sistemas de reservação e distribuição predial na cidade do Rio de Janeiro. Cadernos de Saúde Pública, RJ, 5(3), 284-295, jul/set, 1989.

MOMBA, M. N. B.; KFIR, R.; VNETER, S. N.; CLOETE, T. E. An overview of biofilm formation in distribution systems and its impact on the deterioration of water quality. Water SA Vol. 26 No. 1 January 2000.

MORENO, J. Avaliação e gestão de riscos no controle da qualidade da água em redes de distribuição: estudo de caso. Tese (Doutorado) - Escola de Engenharia de São Carlos da Universidade de São Paulo, São Carlos, 2009.

MOSTAFA, N. G.; MATTA, M. E.; HALIM, H. A. Simulation of Chlorine Decay in Water Distribution Networks Using EPANET - Case Study. Civil and Environmental Research, Vol.3, No.13, 2013.

REALI, M. A. P.; MORUZZI, R. B.; PICANÇO, A. P.; CARVALHO, K. Q. Instalações prediais de água fria. Apostila. São Carlos, 2002.

RUFENER, S.; MAUSEZAHL, D.; MOSLER, H. J.; WEINGARTNER, R. Quality of Drinkingwater at Source and Point-of consumption-Drinking Cup As a High Potential Recontamination Risk: A Field Study in Bolivia. J Health Popul Nutr, 2010 Feb, 28(1):34-41.

SANTOS, D. C. Os sistemas prediais e a promoção da sustentabilidade ambiental. Revista Ambiente Construído, v. 2, n. 4, p. 7-18. Porto Alegre, 2002.

SCURACCHIO, P. A. Qualidade da água utilizada para consumo em escolas no município de São Carlos - SP. Dissertação (Mestrado) - Faculdade de Ciências Farmacêuticas da Universidade Estadual Paulista "Júlio de Mesquita Filho", Araraquara, 2010. 
SCURACCHIO, P. A.; FARACHE FILHO, A. Qualidade da água utilizada para consumo em escolas e creches no município de São Carlos - SP. Alim. Nutr., Araraquara, v. 22, n. 4, p. 641647, out./dez. 2011.

SEMENZA, J. C. et al. Water distribution system and diarrheal disease transmission: a case study in Uzbekistan. Am. J. Trop. Med. Hyg., 59(6), 1998, pp. 941-946.

SILVA, S.R. et al. O cuidado domiciliar com a água de consumo humano e suas implicações na saúde: percepções de moradores em Vitória (ES). Eng Sanit Ambient, v.14 n.4, out/dez 2009, $521-532$.

SWISTOCK, B. R.; CLEMENS, S.; RUMMEL, S. Water Quality and Management of Private Drinking Water Wells in Pennsylvania. Journal of Environmental Health, vol 75, n. 6, January/February 2013.

TOMAZ, P. Aproveitamento de água de chuva em áreas urbanas para fins não potáveis. Apostila. São Paulo, 2010.

TREVETT, A. F.; CARTER, R. C.; TYRREL, S. F. The importance of domestic water quality management in the context of faecal-oral disease transmission. Journal of Water and Health, 03.3, 2005, pp. 259-270.

TREVETT, A. R.; CARTER, R. C.; TYRREL, S. F. Water quality deterioration: a study of household drinking water quality in rural Honduras. International Journal of Environmental Health Research, vol. 14 (4), 2004, pp. 273-283.

TULARAM, G. A.; PROPERJOHN, M. An investigation into modern water distribution network security: risk and implications. Security Journal, n. 24, p. 283-301. 2011.

WRIGHT, J.; GUNDRY, S.; CONROY, R. Household drinking water in developing countries: a systematic review of microbiological contamination between source and point-of-use. Tropical Medicine and International Health. volume 9, no 1, pp 106-117, january 2004. 